\title{
Improving Teaching Materials through Digital Book Reading Log
}

\author{
Zhuo Ren ${ }^{1, \text { a }}$, Noriko Uosaki ${ }^{2}$, Etsuko Kumamoto ${ }^{3}$, \\ Gi-Zen Liư ${ }^{4}$, Chengjiu Yin ${ }^{3, b^{*}}$ \\ ${ }^{1}$ Jinan University, Guangzhou, China; \\ ${ }^{2}$ Osaka University, Osaka, Japan; \\ ${ }^{3}$ Kobe University, Kobe, Japan \\ ${ }^{4}$ Nationnal Cheng Kung University, Taiwan
}

afreeseagull@163.com, ${ }^{b}$ yin@lion.kobe-u.ac.jp

${ }^{*}$ corresponding author

Keywords: Educational Big Data, digital text book, reading logs, teaching assistant tool

\begin{abstract}
Nowadays, it is easy to create digital books. However, through many university teachers create their own digital books as their teaching materials, it is difficult to evaluate and improve. In this paper, based on our previous research on the learning behavior analysis results, we prototype a digital book reading system and analyze the student reading logs and show how teachers can use the results to improve their teaching.
\end{abstract}

\section{Introduction}

Recently, the "Big Data" has become a mainstream in many research fields, and there is no exception in the field of education. With the development of e-publishing technologies and standards, more and more traditional textbooks are being replaced by digital books. By using digital books, a large amount of $\log$ data could be accumulated. It is bringing the changes in the research of education. Many researchers are actually paying attention to the development of digital books, which is used to support teaching, learning and scholarship [1].

Moreover, in the universities, many teachers create and assign their own teaching materials to students based on their own points of views and their own experience without objective evaluation. Especially for the specialized field, many of teachers create their teaching materials through reading some publication papers in their research fields. Therefore, improving teaching materials is a very important issue.

In our previous study, we have analyzed some digital book logs, and found some measures (such as turning to next/previous page, memo, zoom in/out, jumping to a page, adding marker) which have strong correlations with teaching materials. We refer to digital books log entries, such as "turning to the next/previous page," "writing a memo on a page," "jumping to another page," "zooming in/out on a page," and "adding a marker on a page" as "learning behaviors."[2]

Based on the previous study of learning behaviors analysis results, we propose to develop an evidence-based education strategy system to help teachers to improve their teaching materials. This system will visualize the learning behaviors of digital books, and teachers can improve their teaching materials by using the visualization results. The learning behavior log data can provide evidence for improving teaching materials [3].

\section{Literature Review}

Digital books. In the digital age, it is easy to get digital books such as "living books", "talking books", and "CD-ROM books" from internet [2]. Digital books have become a potentially effective 
pedagogic tool [4-6] which is supported by the fact that e-book share significantly increased from $17 \%$ in 2011 to $21 \%$ in 2012 in America [7].

Book reading log. In previous researches, Bakia and Güvelib discussed using book reading logs to evaluate the usage of teaching materials [8]. Ishi and Miwa proposed an approach to improve web-based teaching materials through access logs of students. They used access logs such as students' learning space, students' questions, etc. [3]. However, there is no available research to improve teaching materials by using book reading logs.

Evidence-based education is used to apply the best available evidence gained from the scientific method to educational decision making. "Evidence-based teaching" refers to the teaching aspects [9]. In this paper, we collect the learning behavior data as "evidence that learning has occurred". These evidence then would be used to improve the teaching materials.

\section{Methodology}

Based on the data source, previous studies on data collection could be classified into three categories: Questionnaire-based Data Collection (QDC), Manual Data Collection (MDC), and Automatic Data Collection (ADC). [10]

QDC. In this category, data are collected by using a predesigned questionnaire. Ho et al. (2013) used a questionnaire to investigate the teacher behavior on adopting mobile phone messages as a parent-teacher communication medium [11].

MDC. In this category, a manual data collection system is opened to users, who can employ the system and consciously provide data about their learning behaviors. For example, Chiang et al. (2014) provided an augmented reality (AR) system to guide students in knowledge sharing in inquiry learning activities [12]. In this approach, students capture images from an authentic environment and share these with others.

ADC. In this category, learning behaviors log data are automatically recorded while reading e-documents.

For categories QDC and MDC, the data are consciously collected. Therefore, data are affected by users' own subjective factors. For category ADC, the data is objectively collected, thereby removing the subjective factors that affect data authenticity.

The present work falls under category ADC. By using e-pub format, an web-based digital book system was developed and used for this research (Fig. 1, Fig. 2). Fig. 1 is an interface for students, and Fig. 2 is an interface for teachers. By using this online digital book reading system, we can collect data like "turning to next/previous page", "memo", "zoom in/out", "adding marker". These data were used to analyize learning behaviors.

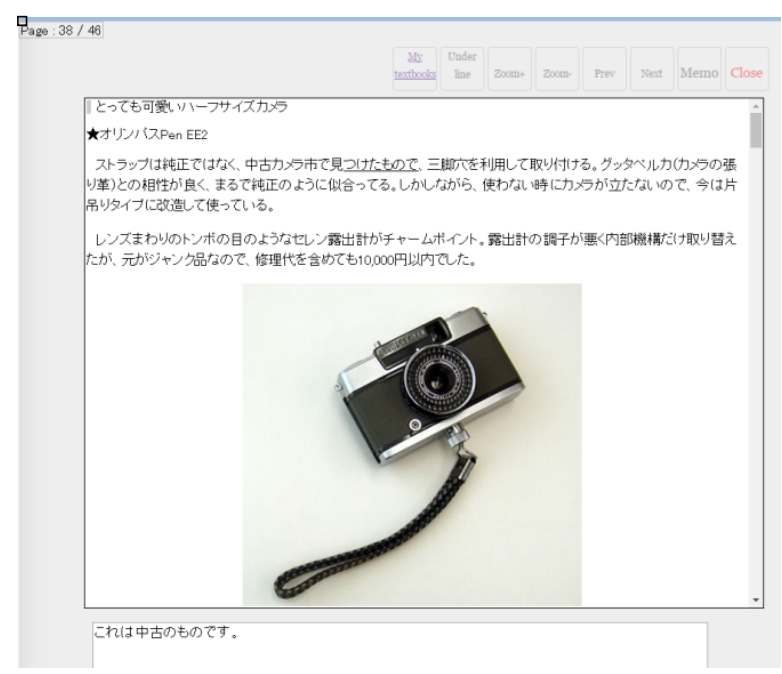

Fig. 1. Student interface

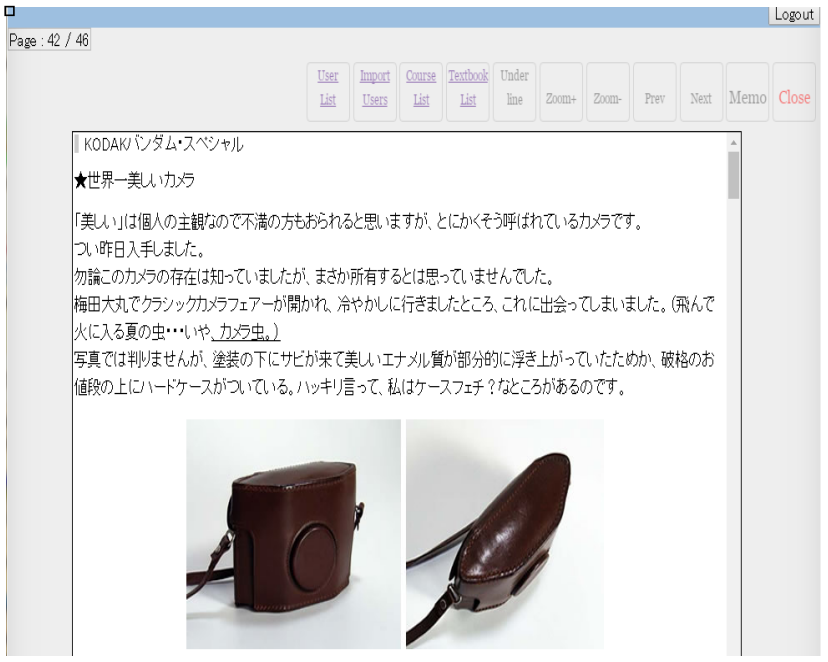

Fig. 2. Teacher interface 
Learning behaviors were analyized in our previous research (Yin, 2015). We found that some learning behaviors such as "Backtrack Reading," "Jump reading", "Zoom", "Marker", and "Memo" have correlation with the teaching material.

Backtrack Reading. We have visualized the students' learning behaviors by using the "Page No," "Prev," and "Next" logs. It was found in the study that a number of students recorded many "Prev" actions, indicating their review of previous pages many times. It is called "Backtrack Reading", which refers to an action to go back to the previous pages while reading. For example, if current knowledge refers to a previously discussed knowledge, they would go back to previous pages for reviewing [2]. One of the main reasons for "Backtrack Reading" is that the content is not easy to be understood; therefore they backtrack to the previous pages frequently. If we provide teachers with the "Backtrack Reading" information, they can confirm the teaching contents and make changes, such as adding more explanations.

Jump Reading. From the visualization of students' learning behaviors, we also found that many students use the "page jump" function, by which they can jump from one page to another page (not the adjacent page). For example, if current page is p.10, then they jump to p.6. This means p.10 has correlation with that of $\mathrm{p} .6$. If we provide teachers with this page jump information, they can improve their teaching materials by changing the order of the pages or adding the link to these two pages.

Zoom. We have analyzed the pages that students always use the zoom in/out function, we've found that these pages have a lot of contents, and students have to read the paper by using zoom in/out. This has brought inconvenience to the students. If we inform the teacher about a number of zoom pages, the teacher can confirm and modify their teaching materials. In/out Zoom could also be frequently used in this textbook if layout has problems.

Marker. Most students use at Marker function at the same place. It shows that the content of this part is very important. The teacher can emphasize the content by highlighting it. That is convenient for students to find the key points.

Memo. Usually the contents that the students make notes on the pages of the textbook are very important. When we inform the teacher of the pages with many notes, the teacher could further improve them by adding more description.

\section{Result: System development}

According to the findings above, we propose a system to improve teaching materials (Fig. 3). There are two parts in this figure. Part A is a visualization of students' reading behaviors, here $\mathrm{P}$ means page, "number" is page number. For example, "P10" means the $10^{\text {th }}$ page. The line among the pages means student from one page go to the another page. Through this figure, we can find that students do "Backtrack Reading" frequently on some pages, for example from P29 to P28, P28 to P27, P27 to P26.

Part B is learning behaviors lists; they are "BACKTRACK LIST", "JUMP LIST", "ZOOM LIST", "MARKER LIST", and "MEMO LIST".

BACKTRACK LIST means how many times that students do "Backtrack reading". In this figure, we can know students do "Backtrack reading" 8 times from P28 to P27, and 21 times from P33 to P32.

JUMP LIST means how many times that students jump from one page to another page. In this figure, we can know that students jump from P24 to P19 one time, and jump from P33 to P26 two times.

ZOOM IN/OUT LIST means how many times that students use the "ZOOM IN" function on one page. In this figure, we can know that students use "ZOOM" on P29 3 times, and on P32 10 times.

MARKER LIST means how many times that students highlight the contents by using "MARKER" function. [line ...] means which line was highlighted. In this figure, we can know that student highlighted the content 10 times from line 2 to line 4 on P9.

MEMO LIST means how many students wrote the same notes on the same page. In this figure, we can know that " $1 \mathrm{~GB}=1024 \mathrm{MB}$ " was written for 5 times on P10. It means that 5 students wrote the same word " $1 \mathrm{~GB}=1024 \mathrm{MB}$ " on $\mathrm{P} 10$. 


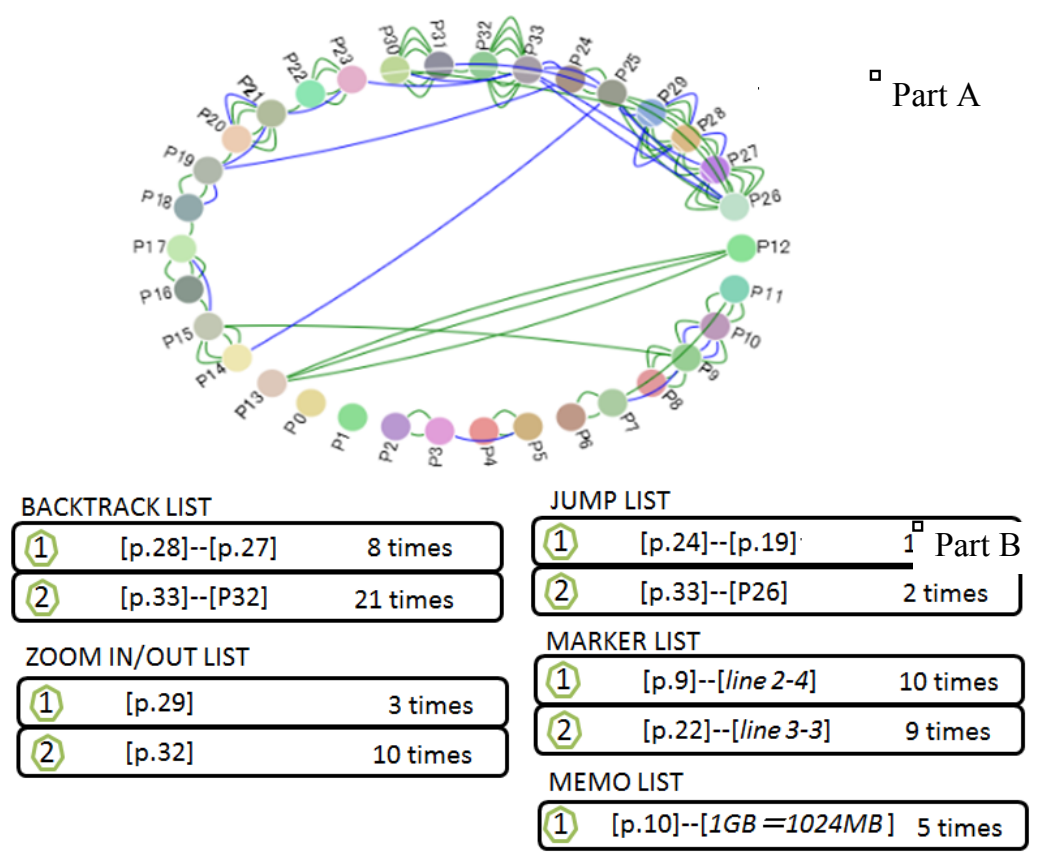

Fig. 3 System

\section{Scenario}

This is a scenario of using this system. The following illustrates in detail how to use our system. The teaching material is created for law course.

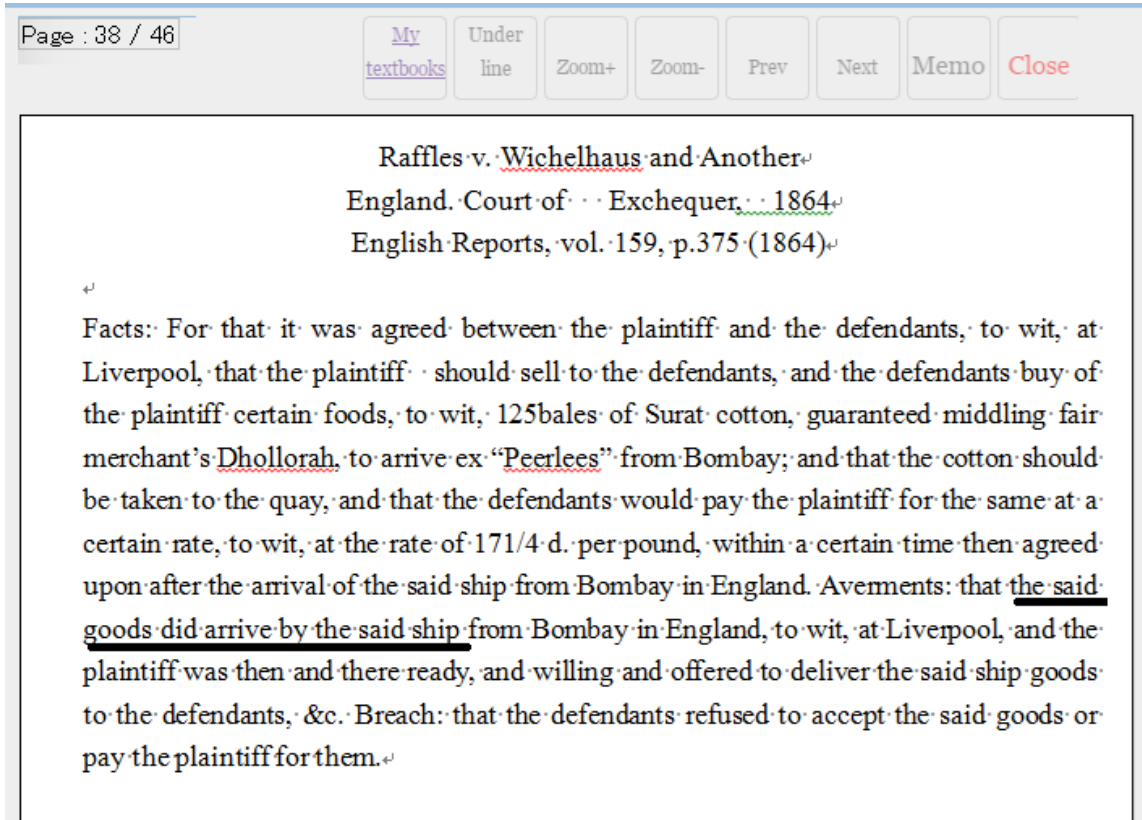

Fig. 4 "Facts" of the case on Page 38 


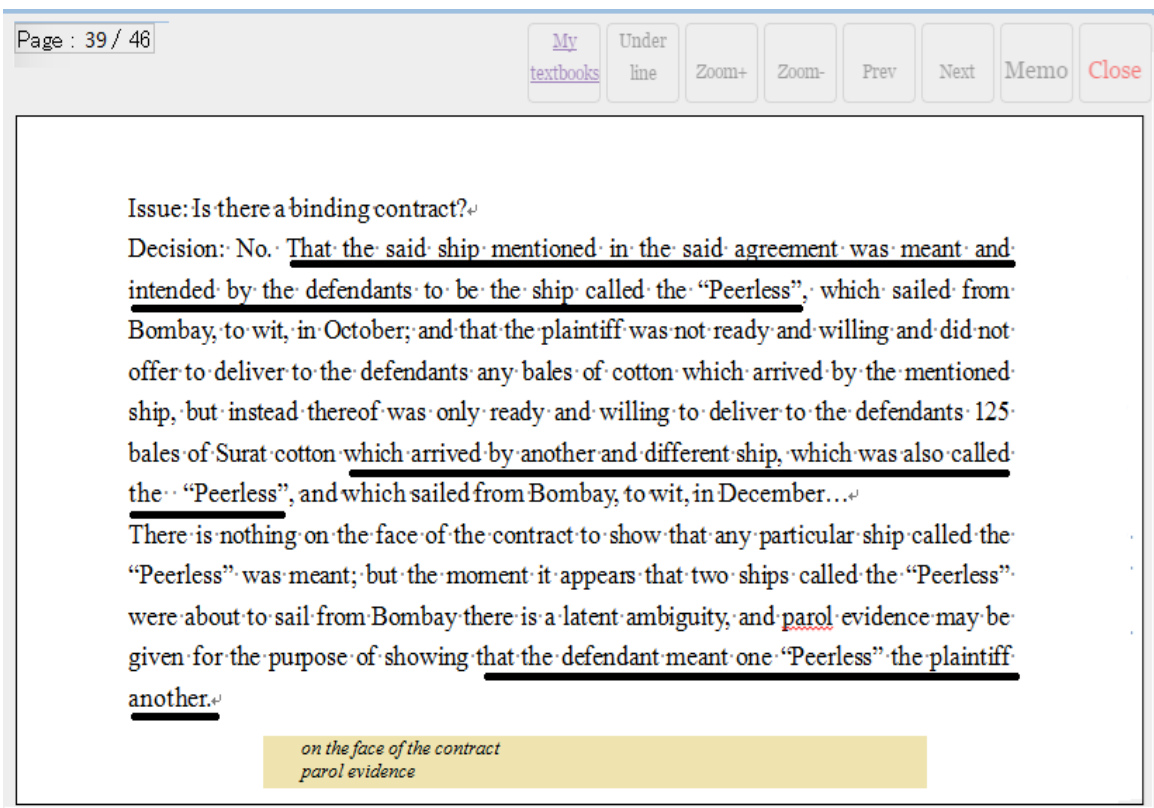

Fig. 5 "Decision" of the case on Page 39

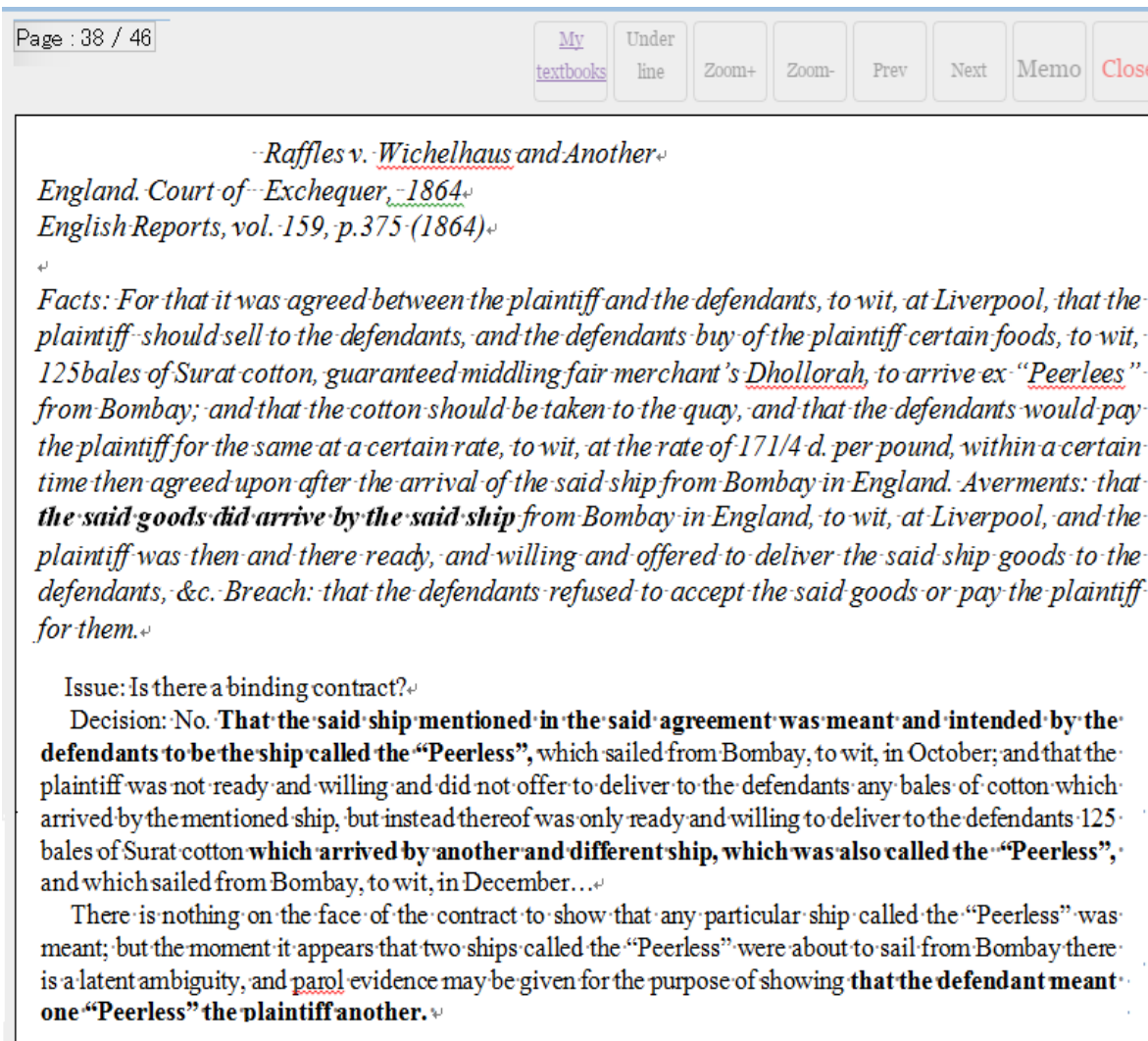

Fig. 6. Making the content into 1 page

At first, we show a case from the course International Business Law to students (Fig. 4, Fig. 5). Fig. 4 is the "Facts" of the case on page 38, and Fig. 5 is the "Decision" of the case on page 39. When the students read the case, we've found that:

1) Students highlight the "the said goods did arrive by the said ship" on the page 38 with "Underline", and highlight some sentences on the page 39 too. According to this, the teacher can highlight these texts by using Bold words (Fig. 6).

2) By analyzing the logs, the leave of many "go to previous page" logs on page 39 , and "go to next page" log on page 38 . This means that they do "Backtrack Reading" frequently. This is because, the 
content of $38^{\text {th }}$ page has correlation with $39^{\text {th }}$ page. Therefore, the teacher can change these two pages into one page (Fig. 6) to make it to be read easily.

3) By analyzing the logs, we found that students jump to the page 30, and page 32 frequently, the applicable theory in this case is called "subjective intent approach", which is explained in the page 30, "Contract Law and CISG" (in page 32) are also relevant with this case. For this case, the teacher can create a link on the system to induce students to the right page.

4) We've found that in the class for this case study, students wrote some notes such as "On the face of the contract" and "Parol Evidence". In this case the teacher can also create a link on the system to induce students to the right page.

\section{Summary}

Many university teachers write their own teaching materials and use them in their lessons. However, many of these teaching materials are usually written based on their own views and their own experiences. How to evaluate these teaching materials and how to improve them are very important research issues.

In this paper, we describe a system which is a very useful teaching assistant tool to support teachers for improving their teaching materials. This system visualizes the learning behaviors and lists up the summary on those learning behaviors based on the reading log data which provide evidence for improving teaching materials.

In the future, we will conduct an evaluation to confirm the learning effectiveness of our system.

\section{Acknowledgements}

Part of this research work was supported by the Grant-in-Aid for Scientific Research No.16H03078 and No.26350319 from the Ministry of Education, Culture, Sports, Science and Technology (MEXT) in Japan.

\section{References}

[1] Yin, C., Okubo, F., Shimada, A., Oi, M., Kojima, K., Yamada, M., Ogata, H. \& Fujimura, N., Smart Phone based Data Collecting System for Analyzing Learning Behaviors, Proc. of the 22nd International Conference on Computers in Education, pp.575-577, Nara, Japan, Nov. 2014.

[2] Yin, C., Okubo, F., Shimada, A., Oi, M., Hirokawa, S., Yamada, M., Kojima, K., \& Ogata, H. (2015), Analyzing the Features of Learning Behaviors of Students using e-Books, 1st workshop on e-Book-based Educational Big Data for Enhancing Teaching and Learning on 23rd International Conference on Computers in Education 2015, pp.617-626, 2015.

[3] Ishii, N. \& Miwa, K., An Evidence Based Approach to Improve Web-based Teaching Materials, Transactions of Japanese Society for Information and Systems in Education, Vol. 25, No. 3, 2008, pp. 292-303.

[4] Hezroni, O. E. (2004). Literacy and assistive technology for children with special needs. Script, 7-8, 195-218, (Hebrew).

[5] Reinking, D. (1997). Me and my hypertext: a multiple digression analysis of technology and literacy (sic). The Reading Teacher, 50, 626-643.

[6] Snyder, I. (2002). Silicon literacies: Communication, innovation and education in the electronic age. London: Routledge.

[7] Rainie, Lee, Kathryn Zickuhr, Kristen Purcell, Mary Madden, and Johanna Brenner (2012). The rise of e-reading. Washington D.C: Pew Research Center's Internet \& American Life Project. http://libraries.pewinternet.org/2012/04/04/the-rise-of-e-reading/

[8] Bakia, E.Güvelib, Evaluation of a web based mathematics teaching material on the subject of functions, Computers \& Education, 51(2),854-863 
[9] Petty, G (2006). Evidence based teaching: A practical approach. Cheltenham: Nelson Thornes

[10] Yin, C., Okubo, F., Shimada, A., Oi, M., Hirokawa, S., Yamada, M., Kojima, K., \& Ogata, H. (2015). Identifying and analyzing the learning behaviors of students using e-books, Proc. of 22nd International Conference on Computers in Education, pp. 118-120.

[11] Ho, L.H., Hung, C.L., \& Chen, H.C. (2013). Using theoretical models to examine the acceptance behavior of mobile phone messaging to enhance parent-teacher interactions, Computers \& Education, vol. 61, pp.105-114.

[12] Chiang, T.H.C., Yang, S.J.H., \& Hwang, G.J. (2014). Students' online interactive patterns in augmented reality-based inquiry activities, Computers \& Education, vol. 78, pp. 97-108. 